\title{
ÉVALUER LES COMPÉTENCES RÉDACTIONNELLES : QUE TESTER ?
}

\author{
Françoise Boch, Julie Sorba, Pascal Bessonneau
}

Armand Colin | « Le français aujourd'hui »

2016/2 N 193 | pages 127 à 144

ISSN 0184-7732

ISBN 9782200930486

Article disponible en ligne à l'adresse :

http://www.cairn.info/revue-le-francais-aujourd-hui-2016-2-page-127.htm

\section{Pour citer cet article :}

Françoise Boch et al., «Évaluer les compétences rédactionnelles : que tester ? 》, Le français aujourd'hui 2016/2 ( $\left.\mathrm{N}^{\circ} 193\right)$, p. 127-144.

DOI 10.3917/lfa.193.0127

Distribution électronique Cairn.info pour Armand Colin.

(C) Armand Colin. Tous droits réservés pour tous pays.

La reproduction ou représentation de cet article, notamment par photocopie, n'est autorisée que dans les limites des conditions générales d'utilisation du site ou, le cas échéant, des conditions générales de la licence souscrite par votre établissement. Toute autre reproduction ou représentation, en tout ou partie, sous quelque forme et de quelque manière que ce soit, est interdite sauf accord préalable et écrit de l'éditeur, en dehors des cas prévus par la législation en vigueur en France. Il est précisé que son stockage dans une base de données est également interdit. 
VARIA

\title{
ÉVALUER LES COMPÉTENCES RÉDACTIONNELLES : QUE TESTER ?
}

\author{
Françoise BOCH \\ Université Grenoble Alpes - LIDILEM \\ Julie SORBA \\ Université Grenoble Alpes - LIDILEM
}

\section{Pascal BESSONNEAU}

Statisticien indépendant

Notre étude s'inscrit dans le cadre d'une recherche appliquée en linguistique et didactique du français visant à valider la fiabilité d'un test mesurant les compétences rédactionnelles des adultes en français (Certificat en compétences rédactionnelles ou $\left.\mathrm{CCR}^{\circledR}\right)$. Pour ce faire, nous avons eu recours à la psychométrie afin de vérifier, d'une part la validité des items choisis, et d'autre part la pertinence de l'architecture du prototype que nous avions conçue. Nous présentons ici le contexte et les choix qui ont présidé à la conception du $\mathrm{CCR}^{\circledR}$, les questions fondant notre problématique, la méthodologie adoptée et les résultats obtenus.

\section{De la nécessité d'un Certificat en compétences rédactionnelles}

Notre interrogation s'appuie sur plusieurs constats convergents. Tout d'abord, d'après l'enquête de $\mathrm{K}$. Schriver auprès d'adultes en situation d'emploi, c'est en moyenne $24 \%$ du temps de travail hebdomadaire qui est consacré à des tâches d'écriture (2012:275). Le recours à l'écrit concerne ainsi tous les secteurs d'activité, y compris les métiers dont l'écriture n'est pas le cœur, et cette pratique est amenée à se développer de manière considérable, selon les prévisions des instituts officiels américains ${ }^{1}$ (Ibid. : 275). À la suite de C. Beaudet et V. Rey (2013) et de B. Labasse (2006), nous qualifierons ces

1. À notre connaissance, peu d'études sont disponibles sur la question de l'évolution des tâches rédactionnelles dans la francophonie. Notons toutefois le rapport S. Lamote et C. Beaudet (2006) qui, à travers leur étude de marché de l'emploi en rédaction professionnelle, souligne la grande variété de ces tâches. Voir également la postface de C. Beaudet (2014), qui relève le caractère fluctuant des genres d'écrits à produire, fortement dépendants des situations professionnelles dans lesquelles ils s'inscrivent. Elle note également le « besoin croissant de spécialistes de l'écrit », face à «l'émergence d'une société de la connaissance, le 
rédacteurs non professionnels de "rédacteurs fonctionnels " pour lesquels écrire n'est pas une profession mais un outil de travail. Ainsi, la plupart des diplômés de l'enseignement supérieur se dirigent vers des professions où ils seront amenés à rédiger efficacement des documents professionnels complexes respectant les conventions de leur milieu et, partant, à se qualifier comme rédacteurs fonctionnels (on peut citer, à titre d'exemple, le cas de l'architecte qui doit répondre à des appels d'offre : l'écriture n'est pas son cœur de métier, mais il en a besoin pour l'exercer). Or, les échos de la presse ${ }^{2}$ comme les entretiens que nous avons menés auprès des recruteurs dans le cadre de notre projet (janvier-juillet 2015) confirment l'inadéquation actuelle entre les compétences réelles des adultes en situation d'emploi et les besoins croissants en entreprise.

En effet, dans l'entreprise, des nouvelles tâches de rédaction apparaissent (par exemple l'animation de réseaux sociaux ou de blog), mais elles ne sont pas - ou plus - prises en charge par des professionnels de la rédaction. Ainsi, quel que soit le secteur professionnel, un nombre croissant de personnes, déjà en poste ou sur le point d'entrer dans le monde du travail, devront assurer ces tâches. Parmi ces personnes, certaines possèdent déjà ces compétences rédactionnelles, du fait de leur expérience ou de leur formation antérieure. La question qui se pose alors est de savoir comment leur permettre de valoriser ces compétences dont l'entreprise a tant besoin aujourd'hui. Pour d'autres qui ne les possèdent pas encore, l'enjeu est de les acquérir pour ensuite les valoriser. C'est pourquoi nous avons conçu et mis en œuvre un Certificat en compétences rédactionnelles permettant à ces publics de valoriser ces compétences, via une formation certifiante.

Le projet Certi-Redac ${ }^{3}$ est ainsi né en 2013 de ces constats convergents. Afin de satisfaire les besoins des différents acteurs professionnels, nous avons tenté d'articuler le point de vue du candidat avec celui du recruteur. Par conséquent, ce certificat poursuit deux objectifs :

- fournir à tout adulte francophone une évaluation fine de son niveau à l'écrit pour l'aider, d'une part, à se positionner et à se former au besoin et, d'autre part, à valoriser ses compétences en milieu professionnel ;

- fournir au recruteur et à l'employeur les moyens d'identifier les compétences à l'écrit de son personnel (actuel ou futur).

développement d'une économie du savoir et de la mémoire, et la transformation des modes de communication" (Ibid. : 203).

2. Voir, par exemple, les articles parus dans Le Figaro du 26 décembre $2014<$ http://etudiant. lefigaro.fr/les-news/actu/detail/article/l-orthographe-preoccupe-les-universites-10340/>, ou encore dans L'Etudiant du 18 mars 2015 <http://www.letudiant.fr/educpros/actualite/ apprendre-l-orthographe-a-l-universite.html>.

3. Le projet Certi-Kedac s inscrit dans le cadire d" un projet de transfert de la recherche académique vers la société civile. La motivation de l'équipe, dirigée par F. Boch et J. Sorba (laboratoire LIDILEM, université Grenoble Alpes, <http://lidilem.u-grenoble3.fr/>, est de mettre à la disposition du grand public un outil utile conçu au sein d'un laboratoire de recherche. Le projet est accompagné sur la partie création de valeur par la LINKSIUM $<$ http://www.linksium.fr/projet/certiredac/>. 
Afin d'offrir un outil fiable aux acteurs opérant dans les métiers du tertiaire, ce certificat est conçu pour évaluer finement les compétences rédactionnelles en français. Labellisé par l'université Grenoble Alpes et issu de la recherche, il comble ainsi un vide dans le domaine de la formation, puisque les tests existants se centrent essentiellement sur l'évaluation de la seule compétence orthographique.

Notre contribution présente donc l'architecture du certificat ; par des tests psychométriques auxquels nous avons soumis notre prototype, elle évalue en outre la pertinence des choix méthodologiques opérés.

\section{Que veut-on tester et pourquoi ?}

Notre hypothèse de travail dans la conception de ce certificat est celle de la transversalité des compétences mises en œuvre dans les écrits professionnels. En effet, même si les écrits professionnels sont variés ( $c f$. Laborde-Milaa et al., dir., 2014) et que la formation doit tenir compte des genres spécifiques aux diverses situations professionnelles, il demeure néanmoins possible de trouver un dénominateur suffisamment commun aux compétences rédactionnelles mises en œuvre dans la majorité des écrits professionnels. Les tâches ciblées dans le certificat ne relèvent donc pas de l'écriture professionnelle spécialisée : ainsi, nous ne testons pas de termes de langue de spécialité ni ne proposons des tâches d'écriture qui relèveraient d'un seul secteur professionnel. En termes d'évaluation, le certificat comporte trois grands objectifs, détaillés ci-dessous, et adopte pour chacun d'eux une méthodologie spécifique.

\section{Identifier les formes et usages corrects dans quatre domaines de compétences}

Ce premier volet du certificat teste les compétences du candidat en orthographe, en lexique, en syntaxe et en ponctuation.

\section{L'orthographe grammaticale}

Nous avons fait le choix de ne tester que l'orthographe grammaticale, et non l'orthographe lexicale. La grande majorité des postes de travail dans le secteur tertiaire étant aujourd'hui pourvus d'ordinateurs, eux-mêmes équipés de correcteurs orthographiques performants, il nous semble en effet superflu de tester les candidats dans ce domaine. En revanche, l'orthographe grammaticale est plus résistante au correcteur informatique, même si les nouveaux modèles sur le marché ${ }^{4}$ ont gagné en efficacité. Au-delà de la question de la qualité de ces outils, ce domaine fait appel à des compétences d'analyse et de logique qu'il est à la fois aisé et intéressant (du point de vue de leur transversalité) de développer dans le cadre de formations ad hoc. Dans cette perspective, ont été sélectionnés pour les items du certificat différents

4. Tels Antidote ou Prolexis par exemple. 
phénomènes morphosyntaxiques (tels que les morphèmes de conjugaison et les accords) dont on a jugé la maitrise utile à la communication écrite. En outre, le choix des items a été effectué à partir des analyses des difficultés des adultes francophones menées par différentes équipes (voir Boch $e t a l$. 2012 ; Rinck et Sitri 2012 ; David 2014).

Plutôt que de procéder par questionnaire à choix multiples (QCM), comme c'est le cas pour la plupart des tests actuellement sur le marché portant sur l'orthographe ${ }^{5}$, nous avons opté pour une dictée lacunaire : au vu des conditions de rédaction dans les situations professionnelles réelles, il nous semble en effet plus pertinent de tester la capacité du candidat à recourir à ses propres stratégies d'analyse (plus ou moins automatisées) pour fournir la forme du mot souhaitée dans un contexte phrastique donné, plutôt que de tester sa capacité à choisir la bonne forme dans une liste fournie.

\section{Le lexique abstrait, transversal et courant}

Rappelons-le : ce certificat se veut transversal aux disciplines et aux métiers. Le lexique testé est donc du lexique à la fois abstrait et courant (extrait de corpus de français contemporain écrit, sur la base d'un critère de fréquence) et concerne aussi bien des substantifs (par ex. prérogatives), des adjectifs (par ex. arbitraire) ou encore des verbes (par ex. réfuter).

\section{La syntaxe (niveaux phrastique et textuel)}

$\mathrm{Au}$ plan syntaxique, les items retenus portent sur des phénomènes linguistiques relevant à la fois de la phrase (par ex. l'usage des pronoms relatifs) ou du texte (par ex. la gestion des pronoms anaphoriques).

\section{La ponctuation : les signes pausaux}

$\mathrm{Au}$ vu de certains travaux disponibles sur la ponctuation auprès d'adultes francophones ( $c f$. notamment Pétillon et Rinck 2014 ; Boch et al. 2015), nous avons choisi, dans le certificat, d'évaluer la gestion des signes pausaux, et en particulier la virgule.

Pour ces trois derniers domaines (lexique, syntaxe et ponctuation), nous avons privilégié le QCM, approprié au type de tâche requise. Afin d'atténuer le poids du hasard inhérent à ce mode de questionnement, et en nous inspirant des travaux menés notamment par D. Leclercq (2006), nous avons inclus systématiquement, dans la liste des réponses possibles fournie au candidat dans les QCM, deux propositions: Toutes les réponses ( $\mathrm{T}$ ») et Aucune réponse ( $\mathrm{A} »)$. L'intérêt de cet ajout est également de favoriser chez le candidat la réflexion et la lecture attentive de toutes les propositions, sans qu'il s'arrête à la première proposition qui lui semble convenir. La figure 1 , ci-dessous, est un exemple extrait de la partie « syntaxe » du certificat

5. Voir, par exemple, le projet Voltaire, qui propose une certification, ou encore la plateforme Orthodidacte, qui offre un test de positionnement. 
qui illustre un cas d'item où la réponse attendue est " aucune réponse " (la forme correcte " auxquels " n'étant pas disponible dans la liste des réponses proposées) :

Consigne : Complétez la phrase avec le ou les mot(s) qui conviennent.

En réponse aux défis l'Afrique doit faire face, il est essentiel que les partenaires du développement accordent la priorité à ce continent.

\begin{tabular}{|l|l|}
\hline 1 & auquel \\
\hline 2 & dont \\
\hline 3 & que \\
\hline 4 & desquels \\
\hline T & Toutes les réponses \\
\hline A & Aucune réponse \\
\hline
\end{tabular}

Tableau 1. : Exemple de question de syntaxe

\section{Produire un texte argumentatif mettant en œuvre différentes compétences}

Le deuxième objectif du certificat est d'évaluer la capacité du candidat à rédiger en situation un texte argumentatif. Il s'agit pour lui, après avoir écouté un court document audio, de rendre compte par écrit et le plus fidèlement possible du point de vue exposé oralement. L'hypothèse de travail qui a guidé notre choix est fondée, ici encore, sur la nature transversale de ce

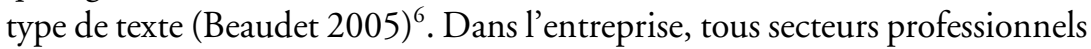
confondus, on peut être amené à rédiger un compte rendu de réunion, un courriel retraçant un entretien téléphonique, ou toute autre tâche requérant l'exposition écrite d'un point de vue autre que le sien. Les critères d'évaluation sont ici sont de trois ordres :

1. Respecter les consignes formelles (longueur attendue, texte rédigé).

2. Produire un texte sémantiquement cohérent (présence des arguments attendus, fidélité par rapport au texte source, etc.).

3. Produire un texte syntaxiquement correct (emploi des collocations en usage ; gestion pertinente de l'anaphore, des pronoms relatifs et de la ponctuation en situation, etc.).

\section{Être lucide par rapport à ses propres compétences}

Le troisième et dernier objectif du certificat est de tester le degré de lucidité du candidat par rapport à ses compétences. En nous appuyant sur les travaux de l'équipe belge du projet Mohican $^{7}$ ( $c f$. notamment Leclercq

6. "En contexte de travail, l'argumentation écrite est incontournable et multiforme " (Beaudet et Clerc $2005: 1$ ).

7. Datant de 2003, le projet de recherche Mohican a consisté à expérimenter un test en Belgique auprès de 3000 étudiants entrant en première année dans neuf universités francophones. 
ibid.), analysant dans le détail l'intérêt pédagogique de cette évaluation (qu'ils nomment pour leur part " degré de certitude»), nous avons intégré cet aspect dans le certificat. Le candidat est systématiquement amené à se prononcer sur la fiabilité qu'il accorde à ses réponses. Ainsi, pour chaque item du certificat (excepté la production écrite, qui se prête mal à ce type d'auto-évaluation métacognitive), le candidat doit indiquer s'il est sûr ou s'il doute de sa réponse.

Avoir une conscience claire de ses compétences a en effet des répercussions importantes en termes d'autonomie dans l'entreprise : pour soi et pour son entourage professionnel, il est sécurisant et efficace de savoir quand s'appuyer de manière solide sur ses propres connaissances et, parallèlement, de savoir quand chercher de l'aide, via des modalités variables (une formation, le recours à un dictionnaire, à un collègue) lorsqu'on est confronté à ses points faibles. Ainsi, le certificat a pour ambition de fournir au candidat, pour chacune des rubriques techniques testées (orthographe, lexique, syntaxe, ponctuation) non seulement un score relatif à ses compétences mais aussi une indication de son degré de lucidité.

L'ensemble des choix présentés ci-dessus repose sur une conception intégrative de l'écrit qui, relevant de plusieurs composantes mises en œuvres simultanément, ne peut en aucun cas se restreindre au seul champ de l'orthographe, et nécessite en outre, on l'a dit, une tâche de production écrite qui place le scripteur en situation. Cela étant, au-delà de cette justification d'ordre théorique, nous avons éprouvé le besoin d'interroger concrètement la pertinence de ces choix.

\section{Les deux volets de la problématique}

\section{Le test évalue-t-il ce qu'il doit évaluer ?}

Une fois le certificat créé, et avant toute analyse plus approfondie, il est nécessaire de s'assurer de son bon fonctionnement, c'est-à-dire que les objectifs poursuivis par les concepteurs, en l'occurrence évaluer les compétences rédactionnelles, sont atteints. Les caractéristiques désirables du certificat sont sa fiabilité et son pouvoir de discrimination entre les candidats. Pour cela, ont été utilisés des outils psychométriques ${ }^{8}$ décrits dans la section suivante ("Opérationnalisation de la recherche»).

\section{Tester différentes composantes de l'écrit... oui mais}

Tester plusieurs composantes de l'écrit au sein d'un certificat destiné à un grand nombre de candidats constitue un choix extrêmement couteux en temps et pose des problèmes redoutables. Pour la partie QCM, le temps d'élaboration des items est en effet considérable et on comprend pourquoi

8. La psychométrie est un domaine de la psychologie s'intéressant à la mesure d'une compétence ou d'un trait latent psychologique. 
les tests existants s'en tiennent à l'orthographe, largement documentée dans les nombreux manuels de référence sur la question. Il est en effet relativement facile de multiplier à l'infini des énoncés comportant des points orthographiques (quasiment identiques du début du collège à l'âge adulte) de différents niveaux de difficultés. En revanche, plus rares sont les ouvrages de référence concernant la syntaxe des adultes francophones (à laquelle nous intégrons ici la ponctuation), différente de celle des enfants. Quant à la rubrique "lexique " du certificat, c'est sans doute la plus complexe à alimenter (car la moins documentée par les travaux disponibles en didactique du français à destination d'adultes), notamment en termes de degré de difficulté, qu'il faut établir au moyen de prétests adressés à des publics variés.

La tâche de production écrite, à laquelle nous tenons à priori le plus (comment peut-on évaluer la capacité d'un individu à rédiger sans le faire rédiger ?), est également très consommatrice de temps : temps d'élaboration du document source, d'identification de critères objectivants, de formation de correcteurs, de correction, d'harmonisation des scores, etc.

$\mathrm{Au}$ vu de ces fortes contraintes (qui ont nécessairement un impact économique important sur le cout du certificat), deux questions s'imposent :

- Faut-il maintenir l'évaluation des compétences en lexique, en syntaxe et en ponctuation ou peut-on s'en tenir à l'orthographe (plus facile à évaluer) ? Autrement dit, un scripteur ayant développé des compétences orthographiques a-t-il nécessairement construit des compétences dans les autres domaines (syntaxe, lexique, ponctuation)?

- Faut-il maintenir une tâche de production écrite (complexe à évaluer et chronophage)? Autrement dit, un scripteur capable d'identifier les formes et les usages corrects dans les domaines techniques de la langue (orthographe, lexique, syntaxe, ponctuation) est-il capable de produire un texte argumentatif satisfaisant? Concrètement, peut-on se passer de la production écrite pour évaluer les compétences rédactionnelles?

\section{Opérationnalisation de la recherche}

Afin d'éclairer avec rigueur les problèmes posés ci-dessus, nous avons fait passer deux versions du certificat à 366 étudiants. Les copies ont été corrigées par une équipe formée par nos soins et ont été soumises à des tests psychométriques ( $c f$. section suivante "Présentation des résultats »). La population testée lors de cette expérimentation est essentiellement âgée de 18 à 21 ans. Mise à part une trentaine d'étudiants de Master, les sujets sont majoritairement des étudiants de niveau Licence (inscrits à l'université ou en Classes préparatoires aux Grandes écoles). Ces étudiants sont composés à 60,5\% de femmes et à 39,5\% d'hommes. Les disciplines universitaires sont relativement variées : $44 \%$ des étudiants sont inscrits en Sciences (filières Biologie, Géographie et Classes préparatoires aux Grandes écoles d'ingénieurs), et $56 \%$ en Sciences humaines (filières Lettres, Sciences du langage, Langues étrangères appliquées et Langues, Littérature 
et Civilisations étrangères). Toutes les passations se sont déroulées dans la région Rhône-Alpes durant les mois de mars et avril 2015. Les tests psychométriques ont pris en compte ces variables pour vérifier la fiabilité du prototype autrement dit :

1. L'unidimensionnalité du CCR $®$ : les items mesurent-ils une composante latente unique qui serait la compétence en français écrit ?

2. La pertinence de chaque item pour la détermination du niveau des candidats.

3. La fiabilité et la cohérence interne du certificat.

4. L'équité du certificat : les résultats obtenus par les candidats dépendentils seulement de leurs compétences en français écrit?

Par ailleurs, au regard de nos préoccupations de recherche relatives à la pertinence de l'architecture du $\mathrm{CCR}^{\circledR}$, deux questions opérationnelles ont été retenues :

1. Les scores obtenus dans le domaine orthographique et dans les autres domaines testés (lexique, syntaxe, ponctuation) sont-ils corrélés ?

2. Les scores obtenus dans ces quatre domaines sont-ils corrélés aux scores obtenus dans la production écrite?

\section{Présentation des résultats}

Pour vérifier la fiabilité du prototype, il s'agit en premier lieu de mesurer la dimensionnalité du certificat. En effet, les items doivent impérativement mesurer une composante latente unique qui serait la compétence en français 9 Réalisée sur chaque version des tâches en QCM, l'analyse est présentée dans les figures 1 et 2 . Sur ces figures, les points correspondent à la variance expliquée par le facteur 1,2 , puis 3 , etc. Les résultats obtenus nous permettent de constater que le premier point est beaucoup plus élevé, ce qui indique que les items forment essentiellement une seule dimension qui représente, par hypothèse, les compétences en français écrit des candidats.

9. Ce type d'hypothèse se vérifie avec une analyse factorielle de la variance commune entre les items qui détermine s'ils sont tous liés à une unique variable latente. Pour ce faire, ont été utilisées les méthodes suggérées par J. Horn (1965) et par W. Zwick et W. Vetter (1984). Elles ont permis de comparer les résultats obtenus au certificat avec les résultats obtenus par simulation. 


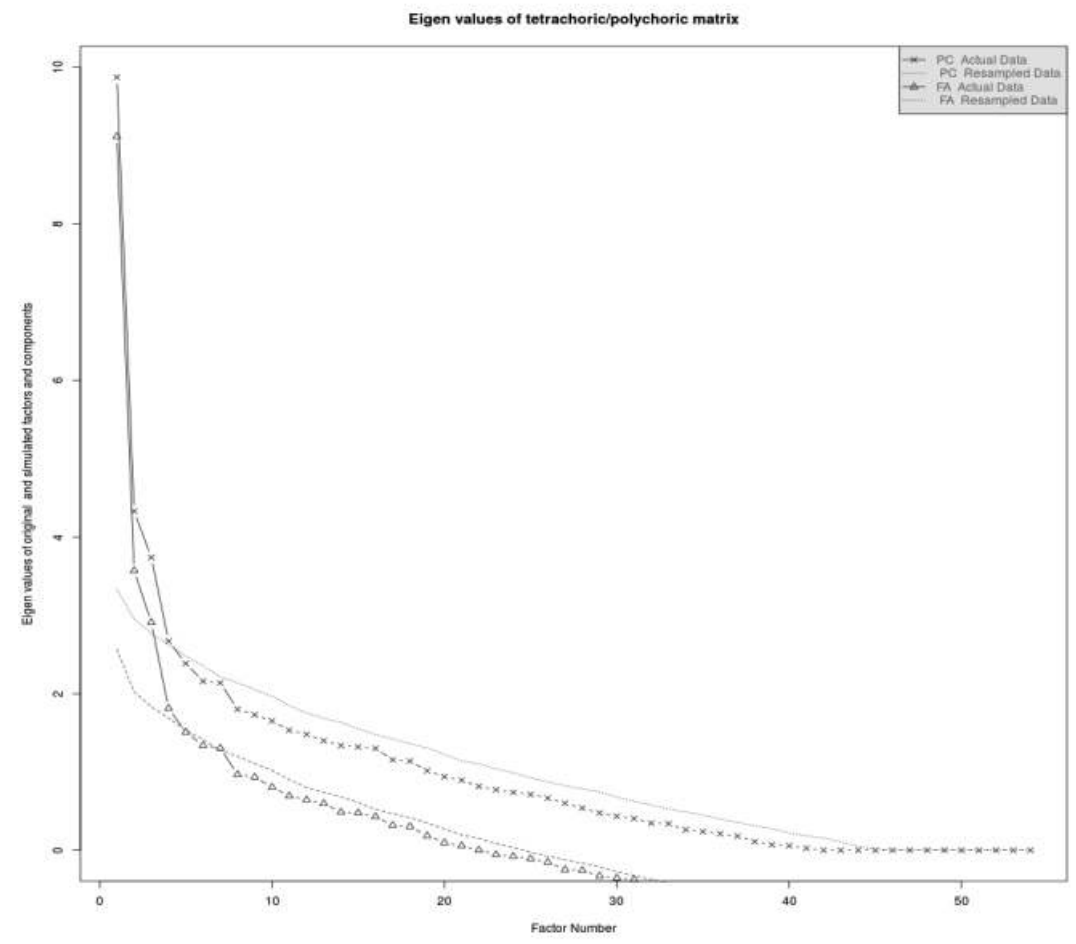

Figure 1. Analyse de la variance en fonction du nombre de facteurs (Version 1)

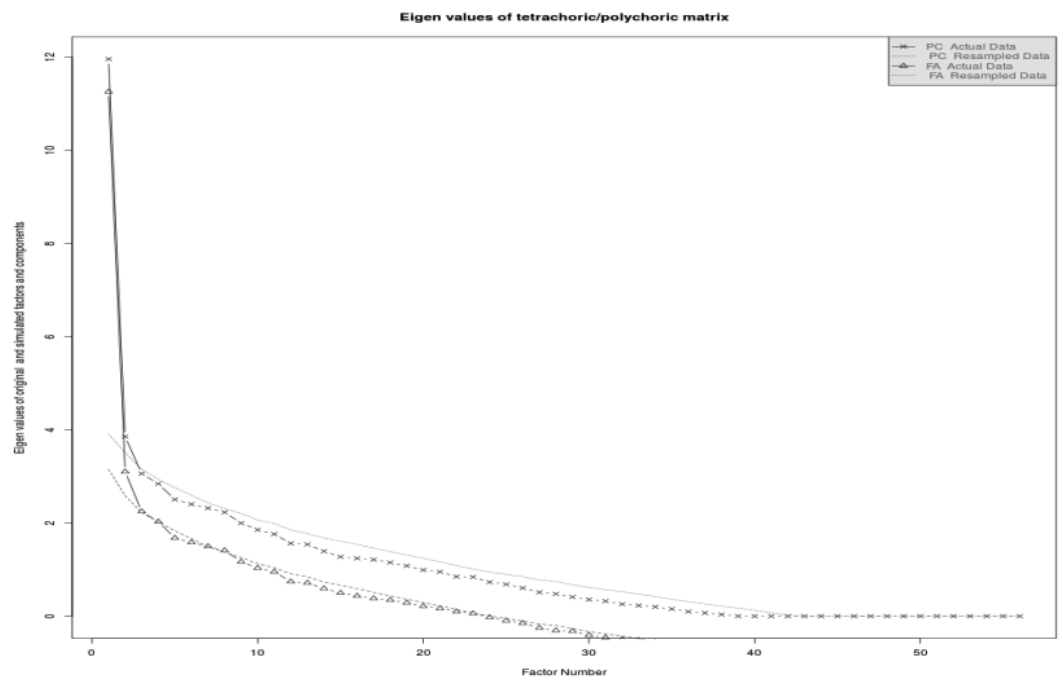

Figure 2. Analyse de la variance en fonction du nombre de facteurs (Version 2) 
Dans un deuxième temps, nous avons vérifié la pertinence de chaque item pour la détermination du niveau des candidats en utilisant les outils décrits dans D. Laveault et J. Grégoire (2002). Il s'agit tout d'abord d'examiner les pourcentages de réussite : sont désirables les items qui ne "saturent " pas, c'est-à-dire qui affichent un taux de réussite entre $95 \%$ et $5 \%$, soit les items qui ne sont pas systématiquement réussis ou échoués. On examine ensuite le coefficient bisérial (Rbis) qui délivre une information sur le pouvoir discriminant de l'item, c'est-à-dire sa capacité à mesurer la compétence des individus ${ }^{10}$. Les résultats obtenus montrent que, dans les versions testées, environ $25 \%$ des items n'ont pas été retenus en raison de leur Rbis. Cette proportion est courante dans les certificats en général et dans les certificats de langue en particulier. Ce processus de sélection permet non seulement d'obtenir un certificat plus fiable, mais aussi d'améliorer le processus de création des items.

On a ensuite procédé à la vérification de la qualité du certificat par l'indice de l'alpha de Cronbac. Pour les deux versions testées, l'alpha de Cronbach est dans l'intervalle de confiance à $95 \%,[0,81 ; 0,88]$ pour la version 1 et $[0,83 ; 0,90]$ pour la version 2 . Cet indice qui varie de 0 à 1 indique la fiabilité et la cohérence interne du certificat. Les résultats obtenus avec des indices de 0,8 sont ici satisfaisants.

Par ailleurs, nous avons également vérifié que le certificat était équitable, c'est-à-dire que les résultats obtenus par les candidats ne dépendaient que de leurs compétences en français écrit. En pratique, la présence de fonctionnements différentiels des items (DIF) se limite aux variables connues. Ainsi, on a vérifié que les items ne présentaient pas de différences de fonctionnement selon les critères de sexe (filles/garçons), de niveau (L1, L2, L3) et de domaine d'études (sciences dites exactes ou sciences humaines). La méthode utilisée pour les DIFs est celle proposée D. Magis et al. (2010). À titre illustratif, les résultats relatifs à ce dernier critère sont présentés dans la figure 3.

10. La borne inférieure de 0,2 est considérée comme un minimum pour retenir les items. 


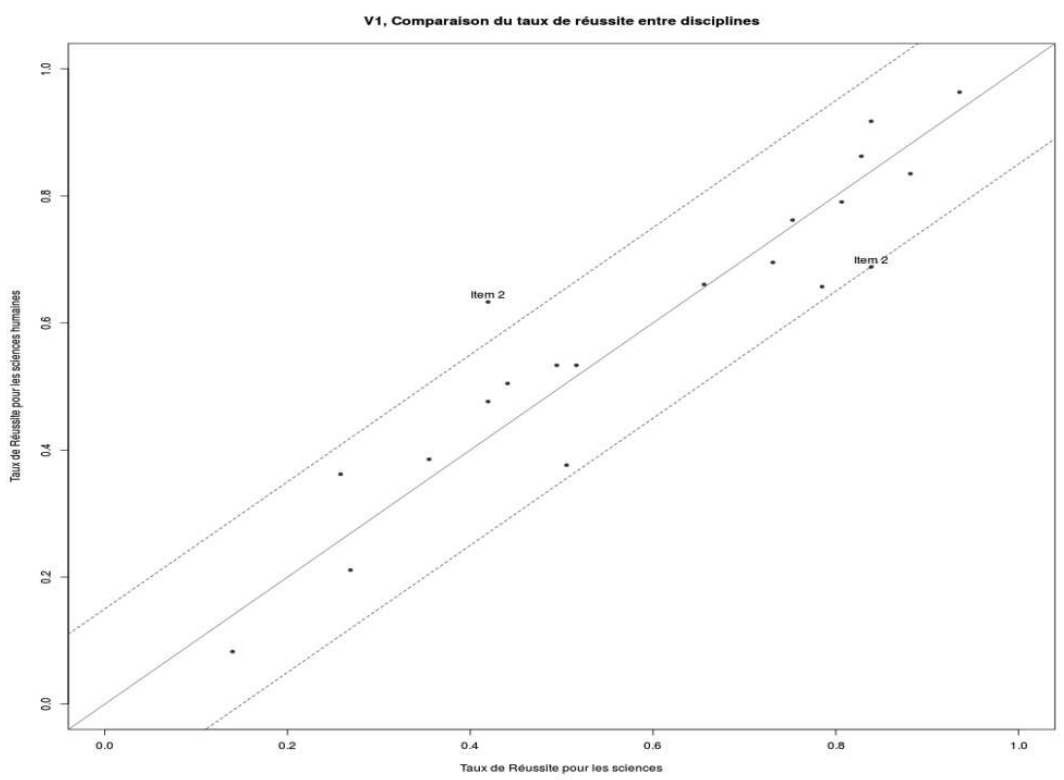

Figure 3. Taux de réussite comparée entre les candidats de sciences dites exactes et ceux de sciences humaines

Dans ce graphique, est comparée la réussite à un item entre deux catégories de candidats (étudiants en sciences humaines $v s$ étudiants en sciences dites exactes). Les items dont la réussite est identique entre les deux groupes sont sur la diagonale. Si des items sont réussis avec des pourcentages différents - comme c'est le cas pour les deux items signalés sur la figure - ces items doivent être revus pour améliorer à la fois le contenu et le processus de création du certificat.

Les outils de la psychométrie permettent également de comparer la difficulté des items et la compétence des candidats, à la condition de disposer d'items en commun entre deux versions du certificat, comme c'est le cas ici. Nous pouvons donc comparer les résultats des candidats ayant passé des versions différentes du certificat, mais également disposer d'une échelle de difficulté pour les items. Le calcul est permis par la théorie de modèle de réponse ${ }^{11}$ à l'item. Sur la figure 4, qui illustre la rubrique " orthographe ", les items (représentés par des croix) sont comparés avec la distribution des compétences (histogramme). Nous pouvons ainsi constater que la difficulté des items correspond relativement bien à la distribution des scores.

11. Dans le cadre des modèles de réponse à l'item simples, à un construit sera associé une variable latente qui permet sur une même échelle de comparer la difficulté des items et la compétence des individus ( $c f$. Bertrand et Blais 2004). 


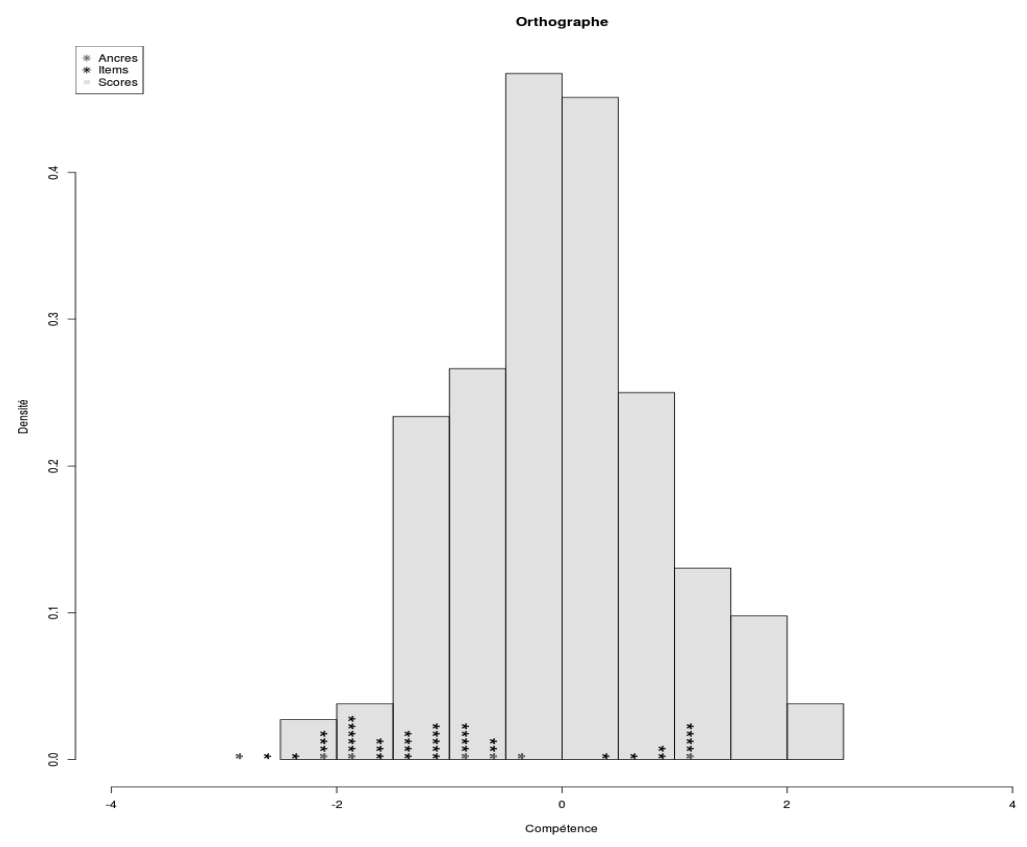

Figure 4. Représentation des items (croix) et de la compétence des candidats (histogramme) pour l'orthographe sur la même échelle

La fiabilité du certificat ayant été établie, nous avons cherché à calculer les corrélations potentielles entre les différentes rubriques du certificat, afin de répondre aux deux préoccupations de recherche relatives à la pertinence de l'architecture du CCR ${ }^{\circledR}$. Les corrélations entre les différentes rubriques du certificat (orthographe, syntaxe, lexique, production écrite) sont représentées graphiquement sur la figure 5 .

On observe que la corrélation des rubriques entre elles est variable. La corrélation est la plus forte pour le couple "orthographe " et "syntaxe " $(0,43)$. Étant donné la distribution des scores pour la ponctuation, l'analyse des résultats est difficile car les résultats sont asymétriquement repartis. La corrélation entre "Production écrite » et le certificat dans son ensemble est de 0,2 : ce faible score témoigne du fait que la production d'écrit et les tests QCM/dictée lacunaire mesurent des compétences sensiblement différentes. 


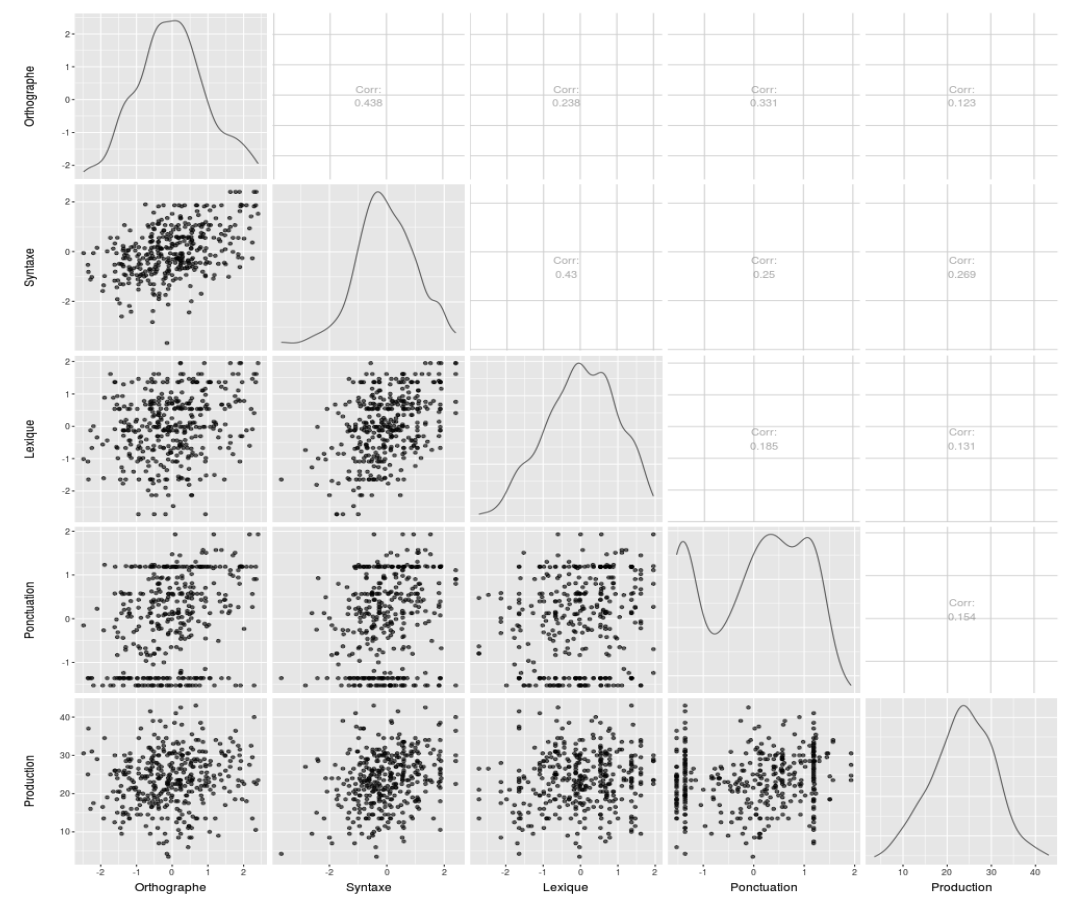

Figure 5. étude des corrélations entre les différentes rubriques du certificat

\section{Conclusion}

Concernant la fiabilité du test, l'analyse de la pertinence des items a fourni des préconisations précises d'amélioration du $\mathrm{CCR}^{\circledR}$ concernant en particulier la suppression des items non discriminants. Les autres analyses psychométriques (unidimensionnalité, fiabilité et cohérence, équité) ont mis en évidence le bon fonctionnement global du $\mathrm{CCR}^{\circledR}$.

Concernant cette fois la pertinence de l'architecture du test, nous avions deux questionnements : le premier portait sur la possibilité de ne conserver dans le certificat que la rubrique "Orthographe ", à l'instar des autres tests sur le marché, et d'évacuer les autres rubriques évaluées par QCM. Or, au vu des résultats obtenus dans le calcul des corrélations, seules les compétences mobilisées dans la tâche relevant de l'orthographe grammaticale semblent corrélées avec celles relevant de la syntaxe, et c'est d'ailleurs plutôt rassurant, les deux domaines étant naturellement très liés : pour produire une forme correcte dans un contexte donné (par exemple, la forme avait dans « j'ai su qu'on vous avait acheté deux billets pour Paris $\left.{ }^{12} »\right)$, il est nécessaire de bien comprendre le fonctionnement syntaxique de la phrase (à savoir, ici, repérer le pronom sujet on, à ne pas confondre avec le pronom complément vous précédant le verbe). Cela étant, la corrélation reste faible, sans doute parce

12. Exemple tiré du certificat. 
que certains phénomènes syntaxiques (telles que les anaphores, dont on sait depuis les années 1980 les difficultés qu'elles posent aux scripteurs ${ }^{13}$ ) ne sont pas réductibles à des questions orthographiques. Ce dernier constat milite en faveur du maintien des deux rubriques " orthographe " et "syntaxe " dans le certificat.

Les deux rubriques "lexique " et "ponctuation ", également évaluées par QCM, apparaissent également indispensables : la première ne présente pas de corrélation avec l'orthographe ${ }^{14}$, tandis que, pour des questions méthodologiques inhérentes à notre expérimentation, on ne sait rien encore de la ponctuation, ce qui ne permet pas à ce stade de l'évacuer. Au-delà de cette limite conjoncturelle, la présence identifiée de la ponctuation dans un certificat en compétences rédactionnelles revêt à nos yeux des vertus pédagogiques, en ce qu'elle favorise la nécessaire prise de conscience de travailler cette dimension de l'écrit, souvent négligée par les scripteurs et, plus largement, par l'institution scolaire (David et Vaudrey-Luigy 2014). En effet, et la recrudescence récente des travaux en linguistique et didactique $\mathrm{du}$ français sur la ponctuation en témoigne ${ }^{15}$, tout scripteur a grand intérêt à en connaitre finement les arcanes pour améliorer sensiblement ses écrits, en particulier au plan de la concision du discours et de la logique dans son exposition. S'entrainer à une bonne gestion des signes de ponctuation afin d'obtenir un meilleur score au certificat présente donc une plusvalue pédagogique : à l'inverse du bachotage, ce type d'entrainement peut avoir des effets bénéfiques à long terme sur la qualité des textes des scripteurs candidats.

Notre deuxième questionnement portait sur la tâche de production d'un texte argumentatif, dont nous nous demandions s'il fallait ou non la conserver dans le certificat. Les résultats sont particulièrement éloquents : la corrélation très faible que les scores obtenus dans ce domaine entretiennent avec les scores obtenus dans les autres rubriques - et encore, uniquement en syntaxe - rend la tâche indispensable. Être capable de produire efficacement un texte argumentatif visant à rendre compte d'un point de vue autre que

13. Voir notamment M.-J. Béguelin (2000), M. Charolles (1995) ou encore B. Combettes (1986), pour ne citer que les travaux les plus connus.

14. Ce résultat remet sérieusement en question l'idée - qui nous semble assez répandue d'une forme de " culture générale linguistique ", qui voudrait qu' on soit « bon » ou « mauvais " à l'écrit, toutes dimensions confondues. Or, il n'y a pas de raison de penser qu'une bonne (ou une faible) connaissance de l'orthographe soit liée à une bonne (ou faible) connaissance lexicale, les compétences mobilisées dans les deux cas étant potentiellement différentes : si l'on sait formellement qu'activité de lecture et acquisition du lexique sont intimement reliées ( $c f$. par exemple Crinon 2011), nous ne disposons pas, à notre connaissance, d'études prouvant une corrélation significative entre activité de lecture et acquisition de l'orthographe. Autrement dit, si, parce que je suis grand lecteur, je dispose d'un répertoire lexical important, rien n'assure que je sache par exemple quelles formes orthographiques prend un participe passé lorsqu'il est conjugué avec être ou avoir.

15. Voir en particulier J. David et S. Vaudrey-Luigy (dir., 2014), ainsi que C. Narjoux (2010) et V. Paolacci et M. Favart (2010). 
le sien fait appel à des compétences spécifiques qui sont manifestement différentes de celles requises dans l'identification de formes correctes au plan orthographique, lexical ou syntaxique.

Ainsi, au sein d'un certificat tel que le nôtre, maintenir l'ensemble des domaines testés constitue une exigence non négociable. Proposer une évaluation à la fois fine et très complète des compétences rédactionnelles s'avère donc complexe et délicat; mais cette difficulté fait aussi la valeur ajoutée du projet et tout son intérêt.

\section{Françoise BOCH, Julie SORBA \& Pascal BESSONNEAU}

\section{Références bibliographiques}

- BEAUDET, C. (2014). Postface. In I. Laborde-Milaa, S. Plane, F. Rinck \& F. Sitri (dir.), Le Discours et la langue, "La formation aux écrits professionnels : des écrits en situation de travail aux dispositifs de formation ", 5(2), 199-204.

- BEAUDET, C. \& CLERC, I. (2005). Argumentation et impact social: le cas des textes utilitaires. Québec : Nota Bene.

- BEAUDET, C. \& REY, V. (2013). Document de cadrage du colloque L'écriture experte, enjeux sociaux et scientifiques, en ligne <http://www.fabula.org/actualites/ 1-ecriture-experte-enjeux-sociaux-scientifiquescolloque-international-universitede-sherbrooke_52568.php>.

- BEGUELIN, M.-J. (2000). Diagnostic des erreurs dans un corpus d'écrits techniques. In M.-J. Béguelin (dir.), La Rédaction technique (pp. 105-119). Bruxelles : De Boeck Supérieur, coll. "Champs linguistiques ».

- BERTRAND, R. \& BLAIS, J.G. (2004). Modèles de mesure : l'apport de la théorie des réponses aux items. Montréal-Québec: Presses universitaires du Québec.

- BOCH, F., BUSON, L. \& BLONDEL, C. (2012). Orthographe \& grammaire à l'université. Quels besoins? Quelles démarches pédagogiques? Scripta, 16(30), 31-51, en ligne sur : <http://periodicos.pucminas.br/index.php/scripta/article/view/ 4238> et sur Diptyque, 24, 139-162.

- BOCH, F., CAVALLA, C., PÉTILLON, S. \& RINCK, F. (2015). Travailler le texte : ponctuation, anaphores, collocations. In F. Boch \& C. Frier (dir.), Écrire dans l'enseignement supérieur : des apports de la recherche aux outils pédagogiques (pp. 53-109). Grenoble : ELLUG.

- CHAROLLES, M. (1995). Comment repêcher les derniers? Analyse des expressions anaphoriques en ce dernier. Pratiques, 85, 89-112.

- CRINON, J. (2011). Lexique et compréhension de textes. In Le Vocabulaire et son enseignement, ressources pour l'école primaire, en ligne site EDUSCOL $<$ http://cache.media.eduscol.education.fr/file/Dossier_vocabulaire/14/6/ Jacques_Crinon_111202_avec_couv_201146.pdf>.

- COMBETTES, B. (1986). Introduction et reprise des éléments d'un texte. Pratiques, 49, 69-84.

- DAVID, J. (2014). Les écarts orthographiques à l'entrée à l'université. Le français aujourd'hui, 185, 95-106.

- DAVID, J. \& VAUDREY-LUIGY, S. (2014). Présentation. Le français aujourd'hui, 187, 3-6. 
- DAVID, J. \& VAUDREY-LUIGY, S. (dir.) (2014). "Enseigner la ponctuation", Le français aujourd'hui, 187. Paris : AFEF \& Armand Colin.

- HORN, J. (1965). A rationale and tests for the numbers of factors in factor analysis. Psychometrika, 30(2), 179-185.

- LABASSE, B. (2006). La Communication écrite. Une matière en quête de substance. Lyon : Éditions Colbert \& CECP Éditions.

- LABORDE-MILAA, I., PLANE, S. \& RINCK, F. (dir.) (2014). "La formation aux écrits professionnels : des écrits en situation de travail». Le Discours et la langue, 5(2).

- LAMOTE, S. \& BEAUDET, C. (2006). Etude du marché de l'emploi en rédaction professionnelle : analyse des offres d'emploi dans trois journaux: Le Devoir, La Presse, Le Soleil, et dans trois sites Internet : Emploi-Québec, Jobboom, Monster, janvier 2005 - juin 2006. Sherbrooke : Université de Sherbrooke, Faculté des lettres et sciences humaines.

- LAVEAULT, D. \& GRÉGOIRE, J. (2002). Introduction aux théories des tests en psychologie et en sciences de l'éducation. Bruxelles : De Boeck.

- LECLERCQ, D. (2003). Analyses psychométriques des questions des 10 checkup MOHICAN : vue d'ensemble. In D. Leclercq (dir.), Diagnostic cognitif et métacognitif au seuil de l'université : le projet MOHICAN mené par les 9 universités de la Communauté française Wallonie-Bruxelles (pp. 173-180). Liège : Éditions de l'université de Liège.

- LECLERCQ, D. (2006). L'évolution des QCM. In G. Figari \& L. Mottier-Lopez (dir.), Recherches sur l'évaluation en Éducation (pp. 139-146). Paris : L'Harmattan. - MAGIS, D., BELAND, S., TUERLINCKX, F., \& DE BOECK, P. (2010). A general frame-work and an R package for the detection of dichotomous differential item functioning. Behavior Research Methods, 42(3), 847-862.

- NARJOUX, C. (2010). La Ponctuation, Règles, exercices et corrigés. Paris : De Boeck-Duculot.

- PAOLACCI, V. \& FAVART, M. (2010). Traitement des marques de cohésion par les jeunes scripteurs : l'utilisation de la ponctuation et des connecteurs à l'entrée en sixième. Approches linguistique, cognitive et didactique. Langages, 177, 113-128. - PÉTILLON, S. \& RINCK, F. (2014). Structure syntaxique et ponctuation : quelques difficultés dans les textes d'étudiants. Colloque Writing Research Across Borders (WRAB III). Université Paris Ouest Nanterre La Défense, 19-22 février 2014.

- RINCK, F. \& SITRI, F. (2012). Pour une formation linguistique aux écrits professionnels. Pratiques, 153-154, 71-84.

- SCHRIVER, K. (2012). What we know about Expertise in Professional Communication. In V. Berninger (dir.), Past, Present, and Future Contributions of Cognitive Writing (pp. 275-312). New York: New York Psychology Press.

- ZWICK, W. \& VETTER, W. (1984). Comparison of five rules for determining the number of components in data sets. Paper presented at the Annual Meeting of the American Psychological Association (92 ${ }^{\text {nd }}$, Toronto, Ontario, August 24-28, 1984). 